\title{
A Semiclassical Condition for Chaos Based on Pesin Theorem
}

\author{
I. Gomez • M. Losada • S. Fortin • M. Castagnino • \\ M. Portesi
}

Received: 9 July 2014 / Accepted: 27 November 2014

(C) Springer Science+Business Media New York 2014

\begin{abstract}
A semiclassical method to determine if the classical limit of a quantum system shows a chaotic behavior or not based on Pesin theorem, is presented. The method is applied to a phenomenological Gamow-type model and it is concluded that in the classical limit the dynamics exhibited by its effective Hamiltonian is chaotic.
\end{abstract}

Keywords Pesin theorem · Lyapunov exponents · Kolmogorov-Sinai entropy · Classical limit

I. Gomez $(\bowtie)$

Instituto de Física de Rosario (IFIR-CONICET), BV. 27 de Febrero 210 Bis Rosario,

Santa Fe, 2000, Argentina

e-mail: stratoignacio@hotmail.com

I. Gomez

Departamento de Física (FCE, Universidad Nacional de La Plata), Instituto de Física La Plata (IFLP),

C.C. 67,1900 , La Plata, Argentina

Present Address:

M. Losada · M. Castagnino

Instituto de Física de Rosario (IFIR-CONICET), BV. 27 de Febrero 210 Bis Rosario,

Santa Fe, 2000, Argentina

M. Losada

e-mail: marcelolosada@yahoo.com

M. Castagnino

e-mail: castagninomario@gmail.com

Present Address:

$\mathrm{S}$. Fortin

CONICET - Departamento de Física, FCEN (UBA), Buenos Aires, Argentina

e-mail: sfortin@gmx.net

Present Address:

M. Portesi

Instituto de Física de La Plata (CONICET-UNLP), Departamento de Física (FCE, Universidad

Nacional de La Plata), C.C. 67, 115 y 49, (1900), La Plata, Argentina

e-mail: portesi@fisica.unlp.edu.ar 


\section{Introduction}

The presence of Lyapunov exponents in quantum systems has been reported in several papers [1-6] and the positiveness of them is a necessary condition for chaos. In classically chaotic quantum systems, decoherence formalism can be used to define quantum chaos. In such case the purity exponentially decreases at a Lyapunov rate [7, 8].

A complete definition of classical chaos can be found in [9], where the three most important features of chaos, Lyapunov exponents, Ergodic Hierarchy $(\mathrm{EH})$ and complexity are studied. Brudno theorem is the link between Kolmogorov-Sinai (KS) entropy and complexity, while Pesin theorem is the link between Lyapunov exponents and KS-entropy as defined in $\mathrm{EH}$ [10].

In this paper we focus on the Pesin theorem, which states that KS-entropy of the system, i.e. the average unpredictability of information of all possible trajectories in the phase space, is equal to the sum of all positive Lyapunov exponents.

A reasonable definition of quantum systems with a chaotic classical description has been given by M. Berry: "A quantum system is chaotic if its classical limit is chaotic" [11]. This quantum caology, as has been named originally by Berry, is what later came to be called quantum chaos.

In previous works $[12,13]$ some of us studied the quantum ergodic hierarchy $(\mathrm{QEH})$. It ranks the chaotic level of quantum systems according to how quantum correlations between states and observables are canceled for large times. From mixing level of QEH we can define a classical statistical limit which allows to reconcile chaos with the Correspondence Principle [14]. In [13] we used QEH to characterize typical chaos phenomena, like the exponential localization of kicked rotator and the quantum interference destruction of Casati-Prosen model in terms of ergodic and mixing levels. Moreover, QEH is an attempt, among several theoretical and phenomenological approaches (like WKB approximation or random matrix theory [15-19]), to define a framework for quantum chaos which admits a chaotic classical description assuming Berry's definition.

In this paper we use QEH idea of ranking quantum chaos with quantum mean values, to present a semiclassical condition for chaos by means of Pesin theorem. More precisely, we express classical quantities by means of quantum mean values, using the Wigner transformation. In particular, we apply this technique to Pesin theorem.

The paper is organized as follows. In Section 2 we present the KS-entropy and Pesin theorem. In Section 3 we review the Wigner transformation, that we employ in the next sections. In Section 4 we express the Pesin theorem by quantum mean values and we obtain a semiclassical condition for chaos that gives a method to determine chaos in the classical limit. In Section 5 we apply this method to a phenomenological Gamow model $[20,21]$ and we conclude that in the classical limit the dynamics exhibited by its effective Hamiltonian is chaotic. Finally, in Section 6 we discuss and draw some conclusions.

\section{Kolmogorov-Sinai Entropy and Pesin Theorem}

We give the general notions of KS-entropy and Pesin theorem within the standard framework of measure theory. We consider a dynamical system $\left(\Gamma, \Sigma, \mu,\left\{T_{t}\right\}_{t \in J}\right)$, where $\Gamma$ is the phase space, $\Sigma$ is a $\sigma$-algebra, $\mu: \Sigma \rightarrow[0,1]$ is a normalized measure and $\left\{T_{t}\right\}_{t \in J}$ is a semigroup of preserving measure transformations. For instance, $T_{t}$ could be the classical Liouville transformation or the corresponding classical transformation associated to the 
quantum Schrödinger transformation. $J$ is usually $\mathbf{R}$ for continuous dynamical systems and $\mathbf{Z}$ for discrete ones.

Let us divide the phase space $\Gamma$ in a partition $Q$ of $m$ small cells $A_{i}$ of measure $\mu\left(A_{i}\right)$. The entropy of $Q$ is defined as

$$
H(Q)=-\sum_{i=1}^{m} \mu\left(A_{i}\right) \log \mu\left(A_{i}\right) .
$$

The KS-entropy of partition $Q$ is given by ${ }^{1}$

$$
h_{\mu}(T, Q)=\lim _{n \rightarrow \infty} \frac{1}{n} H\left(\vee_{j=0}^{n} T^{-j} Q\right) .
$$

From this, the KS-entropy $h_{K S}$ is defined as the supreme of $h_{\mu}(T, Q)$ over all measurable initial partitions of $\Gamma$,

$$
h_{K S}=\sup _{Q} h_{\mu}(T, Q)=\sup _{Q}\left\{\lim _{n \rightarrow \infty} \frac{1}{n} H\left(\vee_{j=0}^{n} T^{-j} Q\right)\right\} .
$$

Moreover, from Brudno theorem it can be proved that KS-entropy is the average unpredictability of information of all possible trajectories in the phase space.

On the other hand, it is well-known that chaos in classical dynamics can be defined by the exponential increase of the distance between two trajectories that start from neighboring initial conditions. Quantitatively, it is related with the largest positive Lyapunov exponent of the system [4]. The positiveness of largest Lyapunov exponent implies exponential instability of motion. In turn, exponential instability of motion is chaotic since almost all trajectories are unpredictable in the sense of information theory.

These two quantities, KS-entropy and the Lyapunov exponents, are related to each other by Pesin theorem, which establishes that [22-24]

$$
h_{K S}=\int_{\Gamma}\left[\sum_{\sigma_{i}(\phi)>0} \sigma_{i}(\phi)\right] d^{2(N+1)} \phi,
$$

where $\sigma_{i}(\phi)$ are the Lyapunov exponents of the dynamical system and $2(\mathrm{~N}+1)$ is de dimension of the phase space. When $\sigma$ is constant over all phase space we have

$$
h_{K S}=\sum_{\sigma>0} \sigma .
$$

At this point, it is appropriate to make a comment on the interest of formula (4) and its precise physical meaning. Pesin theorem relates the KS-entropy, i.e. the average unpredictability of information of all possible trajectories in the phase space, with the exponential instability of motion. Then, the main content of Pesin theorem is that $h_{K S}>0$ is a sufficient condition for chaotic motion.

In Section 4, the condition $h_{K S}>0$ will be used to determine chaos in the classical limit of a quantum system, where $h_{K S}$ will be given by a semiclassical condition in the limit $\hbar \rightarrow 0$.

\footnotetext{
${ }^{1}$ Given two partitions $A$ and $B$ the partition $A \vee B$ is $\left\{a_{i} \cup b_{j}: a_{i} \in A, b_{j} \in B\right\}$. That means $A \vee B$ is a refinement of $A$ and $B$. Given a semigroup of preserving measure transformations $T_{t \in \in J}, T^{-j}$ is the inverse of $T_{j}$, i.e. $T^{-j}=T_{j}^{-1}$.
} 


\section{Weyl-Wigner-Moyal Formalism}

We review the main tools of Wigner transformation for the development of next sections. Given a quantum system we consider its quantum algebra $\mathcal{A}$. If $\hat{f} \in \mathcal{A}$, then the Wigner transformation of $\hat{f}$ is $[25,26]$

$$
f(\phi)=\int_{\mathbf{R}^{N+1}}\langle q+\Delta|\hat{f}| q-\Delta\rangle e^{2 i \frac{p \Delta}{\hbar}} d^{N+1} \Delta,
$$

where $\phi=(q, p) \in \mathbf{R}^{2(N+1)}, p, q, \Delta \in \mathbf{R}^{N+1}$ and $f(\phi)$ is a distribution function over $\mathbf{R}^{2(N+1)}$. From now on we denote $f(\phi)$ by $\operatorname{sym} b(\hat{f})$.

The set of all distribution functions $\mathcal{A}_{q}=\operatorname{symb}(\mathcal{A})$ is called the quasiclassical algebra. Given a pure state $\hat{\rho}_{\psi}=|\psi\rangle\langle\psi|$, its Wigner transformation $\operatorname{symb}\left(\hat{\rho}_{\psi}\right)$ can be negative, then the algebra $\mathcal{A}_{q}$ is not classical. For this reason $\rho(\phi)=\operatorname{symb}(\hat{\rho})$ is called a quasi-probability distribution, where $\hat{\rho}$ is any density matrix of the quantum system.

Given two operators $\hat{f}, \hat{g} \in \mathcal{A}$, we can also introduce the star product [27]

$\operatorname{symb}(\hat{f} \hat{g})=\operatorname{symb}(\hat{f}) * \operatorname{symb}(\hat{g})=(f * g)(\phi)=f(\phi) \exp \left(-\frac{i \hbar}{2} \overleftarrow{\partial}_{a} \omega^{a b} \vec{\partial}_{b}\right) g(\phi)$,

where $f(\phi)=\operatorname{sym}(\hat{f}), g(\phi)=\operatorname{sym}(\hat{g})$ and $\omega^{a b}$ is the metric tensor of the phase space $\Gamma$.

The Moyal bracket is the symbol corresponding to the quantum commutator, i.e.

$$
\{f, g\}_{M B}=\frac{1}{i \hbar}(f * g-g * f)=\operatorname{symb}\left(\frac{1}{i \hbar}[\hat{f}, \hat{g}]\right) .
$$

It can be proved that

$$
(f * g)(\phi)=f(\phi) g(\phi)+\mathcal{O}(\hbar) \quad \text { and } \quad\{f, g\}_{M B}=\{f, g\}_{P B}+\mathcal{O}\left(\hbar^{2}\right),
$$

where

$$
\{f, g\}_{P B}=\sum_{i=1}^{N+1}\left(\frac{\partial f}{\partial q_{i}} \frac{\partial g}{\partial p_{i}}-\frac{\partial f}{\partial p_{i}} \frac{\partial g}{\partial q_{i}}\right)
$$

is the Poisson bracket.

The transformations $s y m b$ and $s y m b^{-1}$ define an isomorphism between the quantum algebra $\mathcal{A}$ and the quasiclassical algebra of distribution functions $\mathcal{A}_{q}$

$$
\text { symb }: \mathcal{A} \rightarrow \mathcal{A}_{q} \quad, \quad \text { symb }{ }^{-1}: \mathcal{A}_{q} \rightarrow \mathcal{A} .
$$

The mapping so defined is the Weyl-Wigner-Moyal symbol. When $\hbar \rightarrow 0, \mathcal{A}_{q}$ tends to $\mathcal{A}_{c l}$, where $\mathcal{A}_{c l}$ is the classical algebra of observables ${ }^{2}$.

A relevant property of the Wigner transformation is [25]

$$
\begin{gathered}
\langle\hat{O}\rangle_{\hat{\rho}}=(\hat{\rho} \mid \hat{O})=\langle\operatorname{symb}(\hat{\rho}), \operatorname{symb}(\hat{O})\rangle=\langle\rho(\phi), O(\phi)\rangle= \\
\int_{\mathbf{R}^{2(N+1)}} d^{2(N+1)} \phi \rho(\phi) O(\phi),
\end{gathered}
$$

where $\langle f, g\rangle$ is the scalar product between $f$ and $g$, and $(\hat{\rho} \mid \hat{O})$ is a notation for the mean value of $\hat{O}$ in $\hat{\rho}$. In other words, $(\hat{\rho} \mid \hat{O})=\langle\hat{O}\rangle_{\hat{\rho}}=\operatorname{tr}(\hat{\rho} \hat{O})$ is the action of the functional $\hat{\rho}$ on the observable $\hat{O}$. Let us make a brief remark about formula (6). It says that the

\footnotetext{
${ }^{2}$ By " $\mathcal{A}_{q}$ tends to $\mathcal{A}_{c l}$ " we mean that in the classical limit, $\hbar \rightarrow 0$, the quassiclassical algebra $\mathcal{A}_{q}$ tends to the commutative algebra of functions defined over $\Gamma$, i.e. $\mathcal{A}_{c l}$, where $\hbar$ is the parameter of deformation quantization.
} 
mean value of an observable $\hat{O}$ in a state $\hat{\rho}$ can be calculated, equivalently, in the quantum algebra $\mathcal{A}$ or in the quasiclassical algebra $\mathcal{A}_{q}$, i.e. as the trace of $\hat{\rho} \hat{O}$ or as the scalar product $\int_{\mathbf{R}^{2(N+1)}} d^{2(N+1)} \phi \rho(\phi) O(\phi)$.

A particular case of (6) is when $\hat{O}$ is the identity $\hat{I}$,

$$
\langle\hat{I}\rangle_{\hat{\rho}}=(\hat{\rho} \mid \hat{I})=\langle\operatorname{symb}(\hat{\rho}), \operatorname{symb}(\hat{I})\rangle=\langle\rho(\phi), I(\phi)\rangle=\int_{\mathbf{R}^{2(N+1)}} d^{2(N+1)} \phi \rho(\phi)=1,
$$

which is nothing but the normalization condition for the state $\hat{\rho}$.

In next section we use the Wigner transformation property given by (6) to express Pesin theorem by means of quantum mean values.

\section{Pesin Theorem Expressed in Terms of Quantum Mean Values: A Semiclassical Condition for Chaos}

With the mathematical background of previous section and the definitions of Section 2 we will write the Pesin theorem in terms of quantum mean values.

As a starting point we make the following assumptions. Let $S$ be a quantum system with its quantum algebra $\mathcal{A}$. We assume $S$ has a classical limit $S_{c l}{ }^{3}$, which is a dynamical system with a phase space $\Gamma$ and a classical group of transformations $\left\{T_{t}\right\}$. Since the process of generating the KS-entropy involves a discrete sequence of steps, the quantum evolution of $S$ is forced to be discretized ${ }^{4}$. We consider $\hat{U}(j)=e^{-i \frac{\hat{H}}{\hbar} \alpha j}$ as the discretized evolution operator $^{5}$ associated with the classical transformation $T_{j}$, where $T$ is taken as $T_{1}$ and the real parameter $\alpha$ defines the time steps.

Then, we show a property that is the key point to express Pesin theorem by means of quantum mean values. Given a partition $Q=\left\{A_{1}, \ldots, A_{m}\right\}$ of $\Gamma$, we write the measure of an element $A_{i}$ of $Q$ at time $t$ as the trace of an appropriate operator $\hat{I}_{A_{i}}$ at time $t$. More precisely, let $I_{A_{i}(t)}(\phi)$ be the characteristic function of $A_{i}(t)$, where $A_{i}(t)=T_{t}\left(A_{i}\right)$ and $A_{i}(t)$ is $A_{i}$ at time $t$. Then, by definition we have

$$
\begin{gathered}
\mu\left(A_{i}(t)\right)=\int d^{2(N+1)} \phi I_{A_{i}(t)}(\phi)=\left\langle I_{A_{i}(t)}(\phi), I(\phi)\right\rangle= \\
\left\langle\operatorname{symb}\left(\hat{I}_{A_{i}}(t)\right), \operatorname{symb}(\hat{I})\right\rangle=\left(\hat{I}_{A_{i}}(t) \mid \hat{I}\right)=\langle\hat{I}\rangle_{\hat{I}_{A_{i}}(t)},
\end{gathered}
$$

where we have used the Wigner transformation property, see (6).

Therefore, $\mu\left(A_{i}(t)\right)=\left(\hat{I}_{A_{i}}(t) \mid \hat{I}\right)$, which means that the measure of $A_{i}$ at time $t$ is equal to the trace of the operator $\hat{I}_{A_{i}}$ at time $t$, where $\hat{I}_{A_{i}}(t)$ is the Wigner transformation of the characteristic function of $A_{i}(t)$.

Next step is to write a semiclassical version $(\hbar \approx 0)$ of the KS-entropy of any partition using the formula (7). Consider a partition $Q=\left\{A_{1}, \ldots, A_{m}\right\}$ of $\Gamma$. Then, we have the partition $B(-n)=\vee_{j=0}^{n} T^{-j} Q$. Let $B\left(k_{0}, k_{1}, \ldots, k_{n}\right)=\bigcap_{j=0}^{n} T^{-j} A_{k_{j}}$ be an element of

\footnotetext{
${ }^{3}$ The classical algebra $\mathcal{A}_{c l}$ of $S_{c l}$ is the limit of the quasiclassical $\mathcal{A}_{q}$ of $S$ when $\hbar \rightarrow 0$, i.e. $\lim _{\hbar \rightarrow 0} \mathcal{A}_{q}=$ $\mathcal{A}_{c l}$.

${ }^{4}$ For instance, discretized evolutions are used in Hamiltonians with a time-dependent potential. In such cases, it is common to take $\hat{U}(n)=\hat{F}(\tau n)$, where $\hat{F}$ is the Floquet operator and $\tau$ is the periodicity of the potential. ${ }^{5}$ In an irreversible process effective Hamiltonians are commonly used to describe open quantum systems, i.e. a quantum system in interaction with its environment. In general, it is not a self-adjoint operator, $\hat{H} \neq \hat{H}^{\dagger}$.
} 
$B(-n)$. Using (22) of Appendix A we can give an expression for $\mu\left(B\left(k_{0}, k_{1}, \ldots, k_{n}\right)\right)$. We have

$$
\mu\left(B\left(k_{0}, k_{1}, \ldots, k_{n}\right)\right)=\left(\prod_{j=0}^{n} \hat{I}_{A_{k_{j}}}(j) \mid \hat{I}\right) \quad \text { when } \hbar \approx 0
$$

that is the trace of the product of operators $\hat{I}_{A_{k_{j}}}(j)$ where $\hat{I}_{A_{k_{j}}}(j)=\hat{U}(j) \hat{I}_{A_{k_{j}}}(0) \hat{U}(j)^{\dagger}$ is $\hat{I}_{A_{k_{j}}}(0)$ after $j$ steps.

Therefore, if we replace $\mu\left(B\left(k_{0}, k_{1}, \ldots, k_{n}\right)\right)$ by $\left(\prod_{j=0}^{n} \hat{I}_{A_{k_{j}}}(j) \mid \hat{I}\right)$ in (2), we obtain

$$
\begin{gathered}
h_{\mu}(T, Q)=\lim _{n \rightarrow \infty} \frac{1}{n} H(B(-n))= \\
-\lim _{n \rightarrow \infty} \frac{1}{n} \sum_{\left(k_{0}, k_{1}, \ldots, k_{n}\right)}^{R_{n}} \mu\left(B\left(k_{0}, k_{1}, \ldots, k_{n}\right)\right) \log \mu\left(B\left(k_{0}, k_{1}, \ldots, k_{n}\right)\right)= \\
-\lim _{n \rightarrow \infty} \frac{1}{n} \sum_{\left(k_{0}, k_{1}, \ldots, k_{n}\right)}^{R_{n}}\left(\prod_{j=0}^{n} \hat{I}_{A_{k_{j}}}(j) \mid \hat{I}\right) \log \left(\prod_{j=0}^{n} \hat{I}_{A_{k_{j}}}(j) \mid \hat{I}\right),
\end{gathered}
$$

where $R_{n}$ is the number of elements of $B(-n)^{6}$.

Then, from (3) and (4), we obtain the Pesin theorem in terms of quantum mean values

$$
\begin{gathered}
\sup _{Q}\left\{-\lim _{n \rightarrow \infty} \frac{1}{n} \sum_{\left(k_{0}, k_{1}, \ldots, k_{n}\right)}^{R_{n}}\left(\prod_{j=0}^{n} \hat{I}_{A_{k_{j}}}(j) \mid \hat{I}\right) \log \left(\prod_{j=0}^{n} \hat{I}_{A_{k_{j}}}(j) \mid \hat{I}\right)\right\} \\
=\int_{\Gamma}\left[\sum_{\sigma_{i}(\phi)>0} \sigma_{i}(\phi)\right] d^{2(N+1)} \phi \quad \text { when } \hbar \approx 0 .
\end{gathered}
$$

Formula (9) implies that if we have a quantum system $S$, with a classical limit $S_{c l}$, the positive Lyapunov exponents of $S_{c l}$ are related with the supreme of an expression which involves the mean values $\left(\prod_{j=0}^{n} \hat{I}_{A_{k_{j}}}(j) \mid \hat{I}\right)$. Moreover, it gives an alternative method for calculating Lyapunov exponents of the classical limit of a quantum system.

As the number $R_{n}$ is usually hard to calculate, usefulness of (9) seems to be restricted to simple cases where $R_{n}$ is trivial. ${ }^{7}$ However, if we are only interested in knowing if $S_{c l}$ is chaotic or not, we do not need to perform the supreme of (9) explicitly. Instead, with the help of the following lemma, it is enough to focus in the asymptotic behavior $(n \rightarrow \infty)$ of $\mu\left(B\left(k_{0}, k_{1}, \ldots, k_{n}\right)\right)$ to ensure the existence of positive Lyapunov exponents and to conclude that $S_{c l}$ is chaotic. The lemma states [22]

$$
\mu\left(B\left(k_{0}, k_{1}, \ldots, k_{n}\right)\right) \text { decreases exponentially } \Longrightarrow K S-\text { entropy }>0 .
$$

This lemma is a sufficient condition for chaos. It says that chaos is governed by the exponential decay of $\mu\left(B\left(k_{0}, k_{1}, \ldots, k_{n}\right)\right)$ in the limit $n \rightarrow \infty$. Physically, this asymptotic limit means looking at the system for large times, without taking into account the details of the chaotic dynamics at finite times, like the formation of fractal structures in a chaotic sea or the

\footnotetext{
${ }^{6} R_{n}$ is well known as the topological entropy of $B(-n)$. Roughly speaking, $R_{n}$ "measures" the degree of mixing of a dynamical system as it evolves in time. Typically, in a fully chaotic system the formation of fractal structures in a chaotic sea can produce numerous sets $B\left(k_{0}, k_{1}, \ldots, k_{n}\right)$ and therefore an increasing of $R_{n}$.

${ }^{7}$ For instance, if $R_{n}$ is uniformly bounded for all $n$, then from (3) it follows that $\sup _{Q}\{\ldots\}=0$. From (4) we obtain $\int_{\Gamma}\left[\sum_{\sigma_{i}(\phi)>0} \sigma_{i}(\phi)\right] d^{2(N+1)} \phi=0$, which implies that $\sigma_{i}(\phi)=0$ for all $i$. Therefore, in such case there is no chaotic behavior.
} 
folding of trajectories. Taking into account that $\mu\left(B\left(k_{0}, k_{1}, \ldots, k_{n}\right)\right)=\left(\prod_{j=0}^{n} \hat{I}_{A_{k_{j}}}(j) \mid \hat{I}\right)$ when $\hbar \approx 0$ (see (8)), then lemma of (10) becomes

$$
\left(\prod_{j=0}^{n} \hat{I}_{A_{k_{j}}}(j) \mid \hat{I}\right) \text { decreases exponentially } \Longrightarrow K S-\text { entropy }>0,
$$

which provides a condition for chaos in the classical limit $S_{c l}$.

Summing up, from the previous steps up to (11) we can obtain a method to determine if the dynamics of $S_{c l}$ is chaotic or not. The prescription of the method is as follows:

(a) Take a generic partition $Q=\left\{A_{i}: i=1, \ldots, m\right\}$ of phase space $\Gamma$ of $S_{c l}$.

(b) For any n-tuple $\left(k_{0}, k_{1}, \ldots, k_{n}\right)$ with $k_{j} \in\{1, \ldots, m\}$ calculate the operators $\hat{I}_{A_{k_{j}}}(j)=\hat{U}(j) \hat{I}_{A_{k_{j}}}(0) \hat{U}(j)^{\dagger}$, where $\hat{I}_{A_{k_{j}}}(0)=\operatorname{sym}^{-1}\left(I_{A_{k_{j}}}(\phi)\right)$.

(c) Then, perform $\left(\prod_{j=0}^{n} \hat{I}_{A_{k_{j}}}(j) \mid \hat{I}\right)$ for all $n$.

(d) Finally, if $\left(\prod_{j=0}^{n} \hat{I}_{A_{k_{j}}}(j) \mid \hat{I}\right)$ decreases exponentially when $n \rightarrow \infty$, then KS-entropy of $S_{c l}$ is positive. Therefore, the dynamics exhibited by $S_{c l}$ is chaotic.

In next section we see how prescription $(a)-(d)$ works with an example.

\section{Physical Relevance}

In order to illustrate the physical relevance of the condition given by (11), we apply the prescription $(a)-(d)$ to an example of the decoherence literature: a phenomenological Gamow model type [20, 21]. This model consists of a single oscillator embedded in an environment composed of a large bath of noninteracting oscillators, which can be considered as a continuum.

The degeneration of this system prevents the application of perturbation theory. Instead, we can apply an analytical extension of the Hamiltonian [21, 28-32] to obtain an non-hermitian effective Hamiltonian $\hat{H}_{e f f}$. Non-hermiticity of $H_{e f f}$ yields two set of eigenvectors $\{\langle\tilde{m}|\}_{m=0}^{\infty}$ and $\{|n\rangle\}_{n=0}^{\infty}$ (left and right eigenvectors, respectively), which satisfy [33]

$$
\begin{gathered}
\hat{H}_{e f f}|n\rangle=z_{n}|n\rangle, \quad\langle\widetilde{n}| H_{e f f}=\langle\widetilde{n}| z_{j}, \quad n \in \mathbf{N}_{0}, \\
\langle\tilde{m} \mid n\rangle=\delta_{m n} \quad(\text { bi } \text { orthogonality }), \\
\sum_{n=0}^{\infty}|n\rangle\langle\tilde{n}|=\hat{I} \quad(\text { completeness }) .
\end{gathered}
$$

The effective Hamiltonian $\hat{H}_{e f f}$ is given by

$$
\hat{H}_{e f f}=\sum_{n=0}^{\infty} z_{n}|n\rangle\langle\widetilde{n}|,
$$

where $z_{n}=n\left(\omega_{0}-i \gamma_{0}\right)$ are complex eigenvalues, except $z_{0}=\omega_{0}, \gamma_{0}$ is associated with the decoherence time $t_{R}=\frac{\hbar}{\gamma_{0}}$ and $\omega_{0}$ is the natural frequency of the single oscillator [20]. 
From formula (23) of Appendix B, we can expand the operators $\hat{I}_{A_{k_{j}}}(j)$ in the bi-orthogonal basis $\{|r\rangle\langle\widetilde{s}|\}_{r, s \in \mathbf{N}_{0}}$

$$
\begin{aligned}
\hat{I}_{A_{k_{j}}}(j)= & \alpha_{A_{k_{j}}}(0,0)|0\rangle\left\langle 0\left|+\sum_{r=1}^{\infty} \alpha_{A_{k_{j}}}(r, r) e^{-2 \frac{\gamma_{0}}{\hbar} r \alpha j}\right| r\right\rangle\langle\widetilde{r}|+ \\
& +\sum_{s=1}^{\infty} \alpha_{A_{k_{j}}}(0, s) e^{i \frac{\omega_{0}}{\hbar}(s-1) \alpha j} e^{-\frac{\gamma_{0}}{\hbar} s \alpha j}|0\rangle\langle\widetilde{s}|+ \\
& +\sum_{r=1}^{\infty} \alpha_{A_{k_{j}}}(r, 0) e^{-i \frac{\omega_{0}}{\hbar}(r-1) \alpha j} e^{-\frac{\gamma_{0}}{\hbar} r \alpha j}|r\rangle\langle 0|+ \\
& +\sum_{r, s>0, r \neq s}^{\infty} \alpha_{A_{k_{j}}}(r, s) e^{-\frac{\gamma_{0}}{\hbar}(r+s) \alpha j}|r\rangle\langle\widetilde{s}| .
\end{aligned}
$$

From (12), we see that for $j \gg \frac{\hbar}{\alpha \gamma_{0}}=\frac{t_{R}}{\alpha}$ all the sums decay exponentially. Then, we can neglect these terms and obtain

$$
\hat{I}_{A_{k_{j}}}(j) \simeq \alpha_{A_{k_{j}}}(0,0)|0\rangle\langle 0| \quad \text { for all } j \gg \frac{t_{R}}{\alpha} \text { with } j=1, \ldots, n .
$$

The coefficient $\frac{t_{R}}{\alpha}$ can be interpreted as an adimensional relaxation time, where parameter $\alpha$ defines the time steps of the discretized evolution.

From (13), we can obtain an asymptotic expression for $\prod_{j=0}^{n} \hat{I}_{A_{k_{j}}}(j)$, when $n \gg \frac{t_{R}}{\alpha}$,

$$
\prod_{j=0}^{n} \hat{I}_{A_{k_{j}}}(j) \simeq\left(\prod_{j=0}^{n} \alpha_{A_{k_{j}}}(0,0)\right)|0\rangle\langle 0| \quad \text { for } n \gg \frac{t_{R}}{\alpha},
$$

and therefore,

$$
\left(\prod_{j=0}^{n} \hat{I}_{A_{k_{j}}}(j) \mid \hat{I}\right) \simeq \prod_{j=0}^{n} \alpha_{A_{k_{j}}}(0,0) \quad \text { for } n \gg \frac{t_{R}}{\alpha} .
$$

Up to (15) we have completed the steps $(a)-(c)$ of our prescription. The last step is to check that $\left(\prod_{j=0}^{n} \hat{I}_{A_{k_{j}}}(j) \mid \hat{I}\right)$ decays exponentially when $n \rightarrow \infty$.

First, we note that when $j \longrightarrow \infty$ we have

$$
\begin{gathered}
\mu\left(A_{k_{j}}(j)\right)=\left(\hat{I}_{A_{k_{j}}}(j) \mid \hat{I}\right)=\alpha_{A_{k_{j}}}(0,0)+ \\
+\sum_{n=1}^{\infty} \alpha_{A_{k_{j}}}(n, n) e^{-2 \frac{\gamma_{0}}{\hbar} n \alpha j} \longrightarrow \alpha_{A_{k_{j}}}(0,0) .
\end{gathered}
$$

Since we consider classical motion is bounded ${ }^{9}$, we can consider phase space $\Gamma$ is normalized. Then, from (16), we have

$$
\mu(\Gamma)=1>\mu\left(A_{k_{j}}(j)\right) \longrightarrow \alpha_{A_{k_{j}}}(0,0) .
$$

\footnotetext{
${ }^{8}$ From (12), it follows that if $n \gg \frac{t_{R}}{\alpha}$, then $\hat{I}_{A_{k_{n}}}(n) \simeq \alpha_{A_{k_{n}}}(0,0)|0\rangle\langle 0|$ is diagonal. Thus, $\prod_{j=0}^{n} \hat{I}_{A_{k_{j}}}(j)=$ $\hat{I}_{A_{k_{0}}}(0) . \hat{I}_{A_{k_{1}}}(1) \ldots \hat{I}_{A_{k_{n}}}(n) \simeq \hat{I}_{A_{k_{0}}}(0) . \hat{I}_{A_{k_{1}}}(1) \ldots \alpha_{A_{k_{n}}}(0,0)|0\rangle\left\langle 0\left|=\left(\prod_{j=0}^{n} \alpha_{A_{k_{j}}}(0,0)\right)\right| 0\right\rangle\langle 0|$ is diagonal, regardless if operators $\hat{I}_{A_{k_{0}}}(0), \hat{I}_{A_{k_{1}}}(1), \ldots, \hat{I}_{A_{k_{n-1}}}(n-1)$ are diagonals or not.

${ }^{9}$ Typically, the phase space of a non-integrable chaotic system is a compact manifold. If motion is regular and integrable, the phase space can be taken as a torus.
} 
Also, given that $\mu\left(A_{k_{j}}(j)\right) \geq 0$ for all $j$, it follows that $0 \leq \alpha_{A_{k_{j}}}(0,0)<1$.

Moreover, if $\mu\left(A_{k_{j_{0}}}\left(j_{0}\right)\right)=0$ for some $k_{j_{0}}$, since $\mu\left(A_{k_{j_{0}}}\left(j_{0}\right)\right)=\left(\prod_{j=0}^{n} \hat{I}_{A_{k_{j_{0}}}}\left(j_{0}\right) \mid \hat{I}\right)$, then $\left(\prod_{j=0}^{n} \hat{I}_{A_{k_{j}}}\left(j_{0}\right) \mid \hat{I}\right)=0$. Therefore, ${ }^{10}$

$$
\left(\prod_{j=0}^{n} \hat{I}_{A_{k_{j_{0}}}}\left(j_{0}\right) \mid \hat{I}\right) \log \left(\prod_{j=0}^{n} \hat{I}_{A_{k_{j_{0}}}}\left(j_{0}\right) \mid \hat{I}\right)=0,
$$

and it does not contribute to the semiclassical version of KS-entropy of (9). This means that we can consider $\mu\left(A_{k_{j}}(j)\right)>0$ for all $j$. Thus, we have

$$
0<\alpha_{A_{k_{j}}}(0,0)<1 \quad \text { for all } j=1, \ldots, n .
$$

If we call $\delta_{1}=\min \left\{\alpha_{A_{k_{j}}}(0,0): k_{j}=1, \ldots, m\right\}$ and $\delta_{2}=\max \left\{\alpha_{A_{k_{j}}}(0,0): k_{j}=\right.$ $1, \ldots, m\}$, then from (19) we have

$$
\delta_{1}^{n+1}<\prod_{j=0}^{n} \alpha_{A_{k_{j}}}(0,0)<\delta_{2}^{n+1} .
$$

Finally, from (15) and (20), we obtain

$$
\delta_{1}^{n+1}<\left(\prod_{j=0}^{n} \hat{I}_{A_{k_{j}}}(j) \mid \hat{I}\right)<\delta_{2}^{n+1} \text { for } n \gg \frac{t_{R}}{\alpha} .
$$

Equation (21) implies that $\left(\prod_{j=0}^{n} \hat{I}_{A_{k_{j}}}(j) \mid \hat{I}\right)$ decreases exponentially. Therefore, by prescription $(a)-(d)$, we conclude the positiveness of Lyapunov exponents of classical limit of the phenomenological Gamow model. Then, in the classical limit the dynamics exhibited by its effective Hamiltonian is chaotic.

\section{Conclusions}

In this paper we used properties of Wigner transformation in order to express classical quantities by means of quantum mean values. In particular, we translated the quantities involved in Pesin theorem and we obtained a version of Pesin theorem expressed in terms of quantum mean values, which relates the Lyapunov exponents of the classical limit of a system with the mean value of the projectors that correspond to characteristic functions on phase space.

Moreover, from the modified version of Pesin theorem, we obtained a method (the prescription $(a)-(d)$ of Section 4$)$ to determine if in the classical limit the dynamics exhibited by the Hamiltonian of a quantum system is chaotic or not. The core of this method is the step $(d)$, which establishes that if we have a quantum system where $\left(\prod_{j=0}^{n} \hat{I}_{A_{k_{j}}}(j) \mid \hat{I}\right)$ exponentially decays when $n \rightarrow \infty$, then in the classical limit the KS-entropy of the quantum system is positive. This also implies that the system must have positive Lyapunov exponents and, therefore, its classical limit must present a chaotic behavior. Summing up,

$$
\left(\prod_{j=0}^{n} \hat{I}_{A_{k_{j}}}(j) \mid \hat{I}\right) \text { exponentially decreases } \begin{array}{r}
\text { in the classical limit the dynamics } \\
\text { exhibited by its Hamiltonian is chaotic }
\end{array}
$$

\footnotetext{
${ }^{10}$ If $f(x)=x \log (x)$, then by definition $f(0)=0$.
} 
Finally, in Section 5, we applied our method to a phenomenological Gamow-type model and we concluded that in the classical limit the dynamics exhibited by its effective Hamiltonian is chaotic. The exponential decay of $\left(\prod_{j=0}^{n} \hat{I}_{A_{k_{j}}}(j) \mid \hat{I}\right)$ occurs for $n>>\frac{t_{R}}{\alpha}$, where $t_{R}$ is the decoherence time and $\alpha$ the real parameter which defines the time steps.

Furthermore, from Pesin theorem expressed in terms of quantum mean values, the quantity $\left(\prod_{j=0}^{n} \hat{I}_{A_{k_{j}}}(j) \mid \hat{I}\right)$ can be related with the positive Lyapunov exponents. This suggests that decoherence time of Gamow-type model could be related with positive Lyapunov exponents of its classical limit. Here we see an interesting hypothesis about a possible relationship between decoherence time and Lyapunov exponents and we hope it will be corroborated in future researches with more examples and theoretical essays.

Acknowledgments This paper was supported partially by the CONICET (National Research Council, Argentina), the IFIR (Instituto de Física de Rosario, Argentina), the IFLP (Instituto de Fsica de La Plata, Argentina) and Universidad de Buenos Aires, Argentina.

The authors would like to acknowledge the anonymous reviewer for helpful comments on the original manuscript.

\section{Appendix A: The Classical Quantity $\mu\left(B\left(k_{0}, k_{1}, \ldots, k_{n}\right)\right)$ Expressed as a Quantum Mean Value}

In order to evaluate the KS entropy, we have to generate the following partition

$$
B(-n)=\bigvee_{j=0}^{n} T^{-j} Q=\left\{\bigcap_{j=0}^{n} T^{-j} A_{k_{j}}: A_{k_{j}} \in Q\right\} \text {, }
$$

If $B\left(k_{0}, k_{1}, \ldots, k_{n}\right)=\bigcap_{j=0}^{n} T^{-j} A_{k_{j}}$ is a generic element of $B(-n)$, then the measure of $B\left(k_{0}, k_{1}, \ldots, k_{n}\right)$ is

$$
\begin{gathered}
\mu\left(B\left(k_{0}, k_{1}, \ldots, k_{n}\right)\right)=\mu\left(\bigcap_{j=0}^{n} T^{-j} A_{k_{j}}\right)=\int_{\bigcap_{j=0}^{n} T^{-j} A_{k_{j}}} d^{2(N+1)} \phi=\int_{\Gamma} I_{\bigcap_{j=0}^{n}}^{n T^{-j} A_{k_{j}}}(\phi) d^{2(N+1)} \phi \\
=\int_{\Gamma} \prod_{j=0}^{n} I_{A_{k_{j}}}\left(T^{j} \phi\right) d^{2(N+1)} \phi=\left\langle\prod_{j=0}^{n} I_{A_{k_{j}}} \circ T^{j}(\phi), I(\phi)\right\rangle=\left\langle\operatorname{symb}\left(\prod_{j=0}^{n} \widehat{I_{A_{k_{j}}} \circ T^{j}}\right), \operatorname{symb}(\hat{I})\right\rangle \\
=\left(\prod_{j=0}^{n} \widehat{I_{A_{k_{j}}} \circ T^{j}} \mid \hat{I}\right)=\left(\prod_{j=0}^{n} \hat{I}_{A_{k_{j}}}(j) \mid \hat{I}\right),
\end{gathered}
$$

where we have used the following properties:

- The characteristic function of an intersection of sets is the product of the characteristic functions of each set.

- If $T$ is bijective, then $I_{T^{-j}} A_{k_{j}}(\phi)=I_{A_{k_{j}}}\left(T^{j} \phi\right)$.

- If $\hbar \approx 0$, then $\operatorname{symb}\left(\prod_{j}^{n} \hat{f}_{j}\right)(\phi) \simeq \prod_{j}^{n} f_{j}(\phi)$, where we have neglected terms of order $\mathcal{O}(\hbar)$ ). This property is the generalization of (5) for a product of $n$ functions $f_{i}$.

- $\widehat{I_{A_{k_{j}}} \circ T} T^{j}=\hat{I}_{A_{k_{j}}}(j)=\hat{U}(j) \hat{I}_{A_{k_{j}}}(0) \hat{U}(j)^{\dagger}$, where $\hat{U}(j)=e^{-\frac{i}{\hbar} \hat{H} \alpha j}$ is the evolution operator and $\alpha$ is a real parameter which defines the time steps. This property is a consequence of the formula (6). 


\section{Appendix B: An Expansion for Operators $\hat{\boldsymbol{I}}_{A_{k_{j}}}$}

We consider a Hamiltonian of the form

$$
\hat{H}=\sum_{r} z_{r}|r\rangle\langle\widetilde{r}|,
$$

where $z_{r}=\operatorname{Re}\left(z_{r}\right)+i \operatorname{Im}\left(z_{r}\right)$ are complex eigenvalues and $\{|r\rangle\},\{\langle\widetilde{s}|\}$ are its two sets of eigenvectors, left and righ respectively [33]. Then we have

$$
\hat{I}_{A_{k_{j}}}(0)=\sum_{r, s} \alpha_{A_{k_{j}}}(r, s)|r\rangle\langle\widetilde{s}| .
$$

Therefore,

$$
\begin{gathered}
\hat{I}_{A_{k_{j}}}(j)=e^{-\frac{i}{\hbar} \hat{H} \alpha j}\left(\sum_{r, s} \alpha_{A_{k_{j}}}(r, s)|r\rangle\langle\tilde{s}|\right) e^{\frac{i}{\hbar} \hat{H}^{\dagger} \alpha j}= \\
e^{-\left(\frac{i}{\hbar} \sum_{p} z_{p}|p\rangle\langle\widetilde{p}|\right) \alpha j}\left(\sum_{r, s} \alpha_{A_{k_{j}}}(r, s)|r\rangle\langle\widetilde{s}|\right) e^{\left(\frac{i}{\hbar} \sum_{q} z_{q}^{*}|q\rangle\langle\widetilde{q}|\right) \alpha j} \\
=\sum_{p} \sum_{q} \alpha_{A_{k_{j}}}(p, q) e^{\left(-\frac{i}{\hbar} z_{p}\right) \alpha j} e^{\left(\frac{i}{\hbar} z_{q}^{*}\right) \alpha j}|p\rangle\langle\widetilde{q}|,
\end{gathered}
$$

where we have used the exponential of an operator $\left(e^{\hat{A}}=\sum_{k=0}^{\infty} \frac{\hat{A}^{k}}{k !}\right)$ and the orthogonal relations of the projectors $|r\rangle\langle\widetilde{s}|$, that is

$$
\begin{gathered}
(|r\rangle\langle\widetilde{r}|)^{k}=|r\rangle\langle\widetilde{r}|, \text { and } \\
\langle\widetilde{s} \mid r\rangle=0 \quad \text { if } \quad r \neq s .
\end{gathered}
$$

\section{References}

1. Alicki, R., Lozinski, A., Pakonski, P., Zyczkowski, K.: Quantum dynamical entropy and decoherence rate. J. Phys. A 37, 5157-5172 (2004)

2. Slomczynski, W., Zyczkowski, K.: Quantum chaos: an entropy approach. J. Math. Phys. 35, 5674-5700 (1994)

3. Slomczynski, W., Zyczkowski, K.: Mean dynamical entropy of quantum maps on the sphere diverges in the semiclassical limit. Phys. Rev. Lett. 80, 1880-1883 (1998)

4. Zyczkowski, K., Wiedemann, H., Slomczynski, W.: How to generalize Lyapunov exponents for quantum mechanics. Vistas Astron. 37, 153-156 (1993)

5. Cucchietti, F.M., Dalvit, D.A.R., Paz, J.P., Zurek, W.H.: Decoherence and the Loschmidt Echo. Phys. Rev. Lett. 91, 210403 (2003)

6. Cucchietti, F.M., Pastawski, H.M., Jalabert, R.A.: Universality of the Lyapunov regime of the Loschmidt echo. Phys. Rev. B 70, 035311 (2004)

7. Monteoliva, D., Paz, J.P.: Decoherence and the Rate of Entropy Production in Chaotic Quantum Systems. Phys. Rev. Lett. 85, 3373 (2000)

8. Monteoliva, D., Paz, J.P.: Decoherence in a classically chaotic quantum system: Entropy production and quantum-classical correspondence. Phys. Rev. E. 64, 056238 (2001)

9. Bellot, G., Earman, J.: Studies in History and Philosophy of Modern Physics. Chaos out of order: Quantum mechanics, the correspondence principle and chaos 28, 147-182 (1997)

10. Berkovitz, J., Frigg, R., Kronz, F.: The Ergodic Hierarchy Randomness and Hamiltonian Chaos. Stud. Hist. Philos. Mod. Phys. 37, 661-691 (2006)

11. Berry, M.: Quantum chaology, not quantum chaos. Phys. Scr. 40, 335-336 (1989)

12. Castagnino, M., Lombardi, O.: Towards a definition of the quantum ergodic hierarchy: Ergodicity and mixing. Phys. A 388, 247-267 (2009)

13. Gomez, I., Castagnino, M.: Towards a definition of the Quantum Ergodic Hierarchy: Kolmogorov and Bernoulli systems. Phys. A 393, 112-131 (2014) 
14. Gomez, I., Castagnino, M.: On the classical limit of quantum mechanics, fundamental graininess and chaos: Compatibility of chaos with the correspondence principle, Chaos. Solitons Fractals 68, 98-113 (2014)

15. Stockmann, H.: Quantum Chaos: An Introduction, page numbers. Cambridge University Press, Cambridge (1999)

16. Haake, F.: Quantum Signatures of Chaos, page numbers. Springer-Verlag, Heidelberg (2001)

17. Gutzwiller, M.C.: Chaos in Classical and Quantum Mechanics, page numbers. Springer Verlag, New York (1990)

18. Casati, G., Chirikov, B.: Quantum Chaos: between order and disorder, page numbers. Cambridge University Press, Cambridge (1995)

19. Tabor, M.: Chaos and Integrability in Nonlinear Dynamics: An Introduction, page numbers. Wiley, New York (1988)

20. Omnès, R.: The Interpretation of Quantum Mechanics, vol. 288. Princeton University, Princeton (1994)

21. Laura, R., Castagnino, M.: Functional approach for quantum systems with continuous spectrum. Phys. Rev. E 57, 3948 (1998)

22. Lichtenberg, A.J., Lieberman, M.A.: Regular and Chaotic Dynamics (Applied Mathematical Sciences), vol. 304. Springer, Berlin (2010)

23. Pesin, Y.: Characteristic exponents and smooth ergodic theory. Russ. Math. Surv. 32, 55-114 (1977)

24. Young, L.: Entropy, p. 283. Princeton University Press, Princeton (2003)

25. Hillery, M., O’Connell, R., Scully, M., Wigner, E.: Distribution functions in physics: Fundamentals. Phys. Rep. 106, 121-167 (1984)

26. Dito, G., Sternheimer, D.: Deformation quantization: genesis, development and metamorphosis. IRMA Lect. Math. Theor. Phys. 1, 9-54 (2002)

27. Bayern, F., Flato, M., Fronsdal, M., Lichnerowicz, A., Sternheimer, D.: Deformation theory and quantization. II, Physical applications. Ann. Phys. 110, 111-151 (1978)

28. Antoniou, I., Suchanecki, Z., Laura, R., Tasaki, S.: Intrinsic irreversibility of quantum systems with diagonal singularity. Phys. A 241, 737-772 (1997)

29. Gadella, M., Pronko, G.: Fortschritte der Physik The Friedrichs model and its use in resonance phenomena, 59, 795-859 (2011)

30. Castagnino, M., Fortin, S.: New bases for a general definition for the moving preferred basis. Mod. Phys. Lett. A 26, 2365-2373 (2011)

31. Ordonez, G., Kim, S.: Complex collective states in a one-dimensional two-atom system. Phys. Rev. A 70, 032702 (2004)

32. Bohm, A.: Quantum mechanics, foundations and applications, pp. 549-563. Springer Verlag, Berlin (1986)

33. Gilary, I., Fleischer, A., Moiseyev, N.: Calculations of time-dependent observables in non-Hermitian quantum mechanics: The problem and a possible solution. Phys. Rev. A 72, 012117 (2005) 